\title{
Incidence of Malocclusions in India - A Review
}

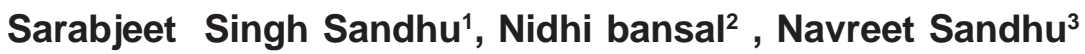

\begin{abstract}
In such a diverse and vast country like India, a large variation in prevalence of malocclusion exists in varying regions of our country. This can be due to variations in ethnicity, nutritional status, religious beliefs, and dietary habits. Various studies have been conducted among diverse Indian regional populations. In this article, a summary of the epidemiological studies is given below.
\end{abstract}

Keywords: Malocclusion, Prevalence, Angle‘s classification, IOTN

${ }^{1}$ Professor and HOD

Dept. of Orthodontics and Dentofacial Orthopaedics Bhojia Dental College and Hospital,

Baddi, Budh Teh. Nalagarh(H.P)

${ }^{2}$ Senior Lecturer

Dept. of Orthodontics and Dentofacial Orthopaedics

Bhojia Dental college and Hospital,

Baddi, Budh Teh. Nalagarh (H.P)

${ }^{3}$ Reader

Department of Prosthodontics ,

National Dental College and Hospital,

Derabassi, Punjab

\author{
Contact Author \\ Dr. Sarabjeet Singh \\ Sarabjeet3400@yahoo.co.in \\ J Oral Health Comm Dent 2012;6(1)21-24
}

\section{INTRODUCTION}

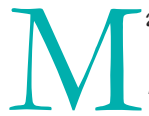

alocclusion is 'any deviation from normal occlusion of teeth. The teeth are in abnormal position in relationship to the basal bone of the alveolar process, to the adjacent teethand/or to the opposing teeth. According to Angle" occlusion is the normal relation of the occlusal inclined planes of the teeth when the jaws are closed"(1).

The methods of recording andmeasuring malocclusion are of two types: a) Qualitative methods were devised mainly for epidemiological studies b) Quantitative (2). Summary of few qualitative methods of recording malocclusion is given as:

ANGLE (1) (1899) devised a classification based on permanent first molar relationship. His method disregarded the relationship of the teeth to the face and although malocclusion was a three dimensional problem, Angle's system had only taken into account antero-posterior deviations in the sagittal plane. When reliability was tested, between examiner errors as well as within examiner errors in categorizing angle class II, division 2 malocclusion were relatively high. Also there was difficulty associated with asymmetry between left and right sides, or those where tooth movements had occurred because of factors such as crowding and premature loss of deciduous teeth (2).
MCCALL(3) (1944): Malocclusion symptoms recorded include: molar relationship, posterior cross- bite, anterior crowding, rotated incisors, excessive overbite, openbite, labial or lingual version, tooth displacement, constriction of arches. No definition of these symptoms was specified. Symptoms were recorded in all or none manner.

WHO/FDI: On the basis of the principles developed for defining andrecording individual traits of malocclusion by Bjork (4) et al, a simplified method was developed during the years 1969-72 working group 2 of FDI commission on classification and statistics for oral conditions. During 1973-7 (5), the method was field tested and modified and published in 1979.The primary objective of the assessment method was to determine the prevalence of malocclusion and dental irregularities and to estimate the treatment needs of the population as a basis for the planning of orthodontic services (6). Five major groups of items were recorded: gross anomalies, dentition (missing teeth, supernumerary teeth ectopic eruption), space dentitions, occlusion, orthodontic treatment need judged subjectively $(5,6)$. However, the method did not define the malocclusion symptoms to be recorded, thus malocclusion symptoms were recorded in all or none manner. Only a few 
malocclusion symptoms were selected arbitrarily as the items to be recorded (2). In this era of evidence based dentistry, quantitative measures are essential, so as to quantitate the treatment and compare our results with some standard of care. Comparing the quality of orthodontic treatment is more difficult. Finding valid measures of treatment success is important for both health care providers and health care consumers. For this several valid and reliable indices have been developed to assess the treatment need and the treatment outcome (2).

Indices have been used to categorize medical and dental disorders for the purpose of epidemiology, research and to allocate patients into categories of treatment need $(8,9,10)$. Index has been defined as a numerical value describing the relative status of a population on a graduated scale with defined upper and lower limits, which is designed to facilitate and permit comparison with other population classified under the same criteria and method. In the orthodontic context, index is used to describe a rating or categorizing system that assigns a numeric score or alphanumeric label to a person's occlusion. However, it does not provide any information concerning the prevalence of given manifestation of malocclusion (6). Several orthodontic indexes have been proposed to provide information on the prevalence of malocclusions and objectively quantify the severity of the various features of malocclusion.

There are five types of indices, each for a distinct purpose. It is the purpose rather than content or conventions of an index that distinguishes it.

- Diagnostic classification: Ex - Angle's classification being used to describe incisor and buccal segment relationships. These classifications serve their purpose reasonably well, allowing ease of communication between orthodontists.

- Epidemiologic indices: Record every trait in a malocclusion to allow estimation of the prevalence of malocclusion in a given population. Ex- Summer's occlusal index, Little's Irregularity index.

- Treatment needs indices: Developed to allow categorization of malocclusion according to the level of treatment need. Ex - Treatment Priority Index, Handicapping malocclusion assessment.

- Treatment outcome indices: Assessment of the outcome of treatment or the changes resulting from treatment. PAR index has been developed specifically for this purpose.

- Treatment complexity Index: Index of Complexity, Outcome and Need (10).

There is ethnic, geographical prevalence of malocclusion. It is more prevalent in whites than in blacks, more in developed countries than in third world countries; more in urban areas than in rural children.

In such a diverse and vast country like India, a large variation in prevalence of malocclusion exists in varying regions of our country. This can be due to variations in ethnicity, nutritional status, religious beliefs, and dietary habits. The prevalence of malocclusion in India varies from 20 $43 \%(11)$.

\section{MALOCCLUSION IN SOUTH INDIA}

The prevalence of malocclusion in southern Indian city of Thiruvananthapuram in age group $12-15$ was reported as $49.2 \%$. Of this, class I malocclusion was 44\%, Class II 4.9\% and class III 0.3\% (12).

Prasad (13) et al (1971) conducted a study in 1033 subjects from Bangalore of age group 5-15 years and reported $51.5 \%$ incidence of Malocclusion. Of this, Class I Malocclusion was 95\%, Class II 4.0\%, Class III $0.9 \%$, crowding $22.0 \%$

NagaRaja Rao (14)(1980) reported a prevalence of Class I malocclusion $23.0 \%$, Class II $4.5 \%$, Class III $1.3 \%$ in 511 (Udupi) subjects ranging from the age 5-15 years. According to another study from Bangalore conducted on 1001 school children aged 12-15 years the prevalence of malocclusion was $49.2 \%$. Prevalence of Class II malocclusion was $4.9 \%$ and class III was $0.3 \%$ (16).
Das et al (16), who conducted an epidemiological study of malocclusion in Bangalore in 2008, reported $71 \%$ of prevalence of malocclusion while Sindhu et al reported a prevalence rate of $90 \%$ in age group 6-30yrs. This is similar to the findings of Prasad et al who conducted an epidemiological study of malocclusion in the age group of 5-15yrs in Bangalore city in 1971, reported a high incidence of malocclusion of $85.7 \%$ with $51.5 \%$ Class I, 4\% Class II, $0.9 \%$ Class III. This is in disagreement with Kharbanda (21) et al who found $36.6 \%$ prevalence of malocclusion in Delhi.

Shivakumar (17) et al did a descriptive cross sectional study among 1000 in the age group 12 to 15 year old school children studying in middle and high schools of Davangere city, India. The author also stated that the DAI appears to be the easiest to use and it does not take into account buccal cross bite, posterior openbite, central line discrepancies or a deep overbite, these factors may have considerable impact on treatment complexity and therefore weakens the index. Malocclusion is not only a single entity but rather a collation of situations each in itself constituting a problem and any of the situations are complicated by a multiplicity of genetic and environmental causes. Further more emphasis should be given on proper preventive and interceptive orthodontic services to the affected group.

Suma (18) et al studied the prevalence of spacing and crowding in 1000 school going urban Bangalore children (below 6yrs). $81 \%$ had spaced dentition, $15.3 \%$ and $4 \%$ children had closed dentition and crowding respectively. Very few children had few dental abnormalities such as flourosis, fusion of teeth. The authors also reported that the frequency of developing malocclusion was more in females than in males as crowding was seen more in females than in males.

\section{MALOCCLUSION IN NORTH INDIA}

Shourie KL (1942), Miglani (1963), Tiwari A (1965) studied the prevalence of malocclusion in mixed dentition stages 
Punjabi subjects and reported a prevalence rate of malocclusion 19.6-37.52\% (19).

Joshi (20) et al studied the primary dentition in 100 children 3-6yrs from Gujarat and reported that spaced dentition was more common than the closed type. They found that amount of spacing was greater in males.

Kharbanda et al $(21,22)$ (1995) reported prevalence of malocclusion in Delhi based on the school survey of 4500 children in the age group of 5-13 years. The sample size was calculated based on sampling design to represent entire school going population of Delhi in three subdivided locations. i.e. urban, periurban and rural. Two types of schools were considered the Convent and Government. The sample data were essentially presented in two major groups: the mixed dentition group which comprised 2817 school children in the age group of 5-9 years and the late mixed/ permanent dentition groups, in the age group 10-13 yea years comprised of 2737 children. In age group 5-9 years: A majority of Delhi children exhibited class I molar relation $(91.6 \%)$, while only' $6 \%$ exhibited class II molar relation. The permanent molar relation was in accordance with deciduous molar relation that was mesial step $(90.3 \%)$ and distal step (8.6\%). Crowding in mandibular anterior teeth was the most common trait of malocclusion (11.7\%). There was no difference in the prevalence of malocclusion between males and females $(23,24)$.

In age group 10-13 years: The prevalence of malocclusion and its traits was $45.7 \%$. This comprised of Class I $27.7 \%$, class II $14.6 \%$ and class III 3.4\% malocclusion. Full cusp Class III malocclusion was only $0.2 \%$. The crowding of anterior teeth in maxilla and mandible was $9.5 \%$ and $18 \%$, respectively. Superior protrusion was $12 \%$ and so was the deep bite. There was no sex affiliation for the prevalence of malocclusion. However, the crowding in maxillary anterior teeth was high among girls $(23,24)$.

The prevalence of malocclusion in rural children in Haryana in the age group 12-16 was found to be $55.3 \%$. Of this, class I malocclusion was 43.6\%, Class 11 9.8\%, class III $0.6 \%$, bimaxillary protrusion $0.5 \%$ and mutilations $0.8 \%$ (25). Jalili, Sidhu and Kharbanda (26) (1995) surveyed 1085 tribal children of 6-14 years of age living in remote villages of Mandu in Madhya Pradesh. The tribal children exhibited a very low prevalence of malocclusion and its traits, as compared to the urban Indian children. Majority of them $(85.6 \%)$ were free from any anomalies of occlusion. The prevalence of malocclusion was only $14.4 \%$. A majority of these $(10.5 \%)$ were of mild malocclusion and a smaller number $(3.7 \%)$ had moderate to severe malocclusion. The 'handicapping malocclusion' was observed in $0.2 \%$ only. The prevalence of distomolar (class II) relationship was $3.8 \%$ of which full cusp distoclusion was $0.6 \%$ only. The overjet and overbite were $0.4 \%$ and $0.3 \%$. The crowding of anterior teeth in the maxillary arch was $6.4 \%$ and in the mandibular arch was $7.8 \%$. From the results of the study, it can be concluded that $80.1 \%$ of school children had little or no malocclusion requiring no or little orthodontic treatment need, $19.9 \%$ of school children had malocclusion ranging from definite to handicapping malocclusion requiring elective to mandatory type of orthodontic treatment.

There is a definite ethnic trend in the prevalence of type of malocclusion in India from north to south of India (19). The prevalence of class II malocclusion in Bangalore and Tiruvananthapuram is reported close to $5 \%$ which is much low compared to the $10-15 \%$ class II malocclusion in Delhi and Haryana. In addition, the southern population has ethnic affinity for bimaxillary protrusion.

In 700 subjects of age range 16-25yrs in Jaipur, Trehan et al (27) reported $66.7 \%$ of subjects had malocclusion and only $33.3 \%$ had normal occlusion. Prevalence of Angle's class I malocclusion was the highest $(57.9 \%)$ and that of Angle's class III malocclusion was the least $(1.4 \%)$. The prevalence of Angle's class II division 2 and Angle's class III malocclusion was low, i.e. $1.9 \%$ and $1.4 \%$ respectively. This is to similar to findings of Singh et al who reported prevalence of Angle's Class II division 2 malocclusion of $5.8 \%$ and Angle's Class III malocclusion of $3.17 \%$ in his study of distribution among North Indians seeking orthodontic treatment. 32.8\% of the males had normal occlusion while Angle's class II division 2 malocclusion was the least prevalent (1.1\%). 35.5\% of the females had normal occlusion while prevalence of Class III malocclusion was the least. There was no statistically significant gender difference in prevalence of malocclusion.

Khandelwal (28) et al reported an incidence of class I (69.15\%), Class II division 1(18.9\%), Class 1 division2 $(27.69 \%)$ class III (3.98\%) in 201 males subject hailing from Indore.

The treatment needs of a society cannot be known from the data on the prevalence of malocclusion alone. Mere existence of a dental irregularity like a diastema or rotation of a tooth may not warrant orthodontic treatment until there is a concern for it. The concerns for the similar type of dental deformity may be least for one individual while it may cause anxiety in another. The same dental malocclusion may be of no significance in an individual at a given age but may be of great concern at a different point of time/age. The need for the orthodontic treatment is also governed by the dental health component (DHC) of malocclusion which is measured in terms of its effect on the longevity dentition and function of the occlusion. Malocclusion like a severe deep bite in a class II division 2 malocclusion may be of least aesthetic concern to an individual but would require a priority in treatment because of its detrimental effect on the dentition.

To prioritize malocclusion for the treatment point of view, many indices have been developed especially in Europe of which IOTN is the widely used. The IOTN may not provide a true picture of orthodontic needs for a country like India for its limitation in recording bimaxillary protrusion. The bimaxillary protrusion which is preva- 
lent in India especially in southern India should be added to the existing recording proforma. In addition, the dental aesthetic index should also be modified to include bimaxillary protrusion. We do not have a single study in the country to point out the data-based treatment needs of India/ state or a city (19).

Pitfalls: Orthodontic indexes have enabled us to quantify the need for treatment; however, they are not always comparable. This is particularly important when comparing the previous epidemiologic studies with different orthodontic indexes. An index could be precise but biased. In such a case, the score will be reproducible but not an accurate portrayal of the occlusion. There seems to be no universally accepted index for measuring malocclusion. Further research would therefore be needed to develop better indices or to refine the present indices so that they can be more universally accepted (29). Many of the current indices of treatment standards show low intra and inter examiner variability when compared with those used for diagnostic or epidemiological purposes

\section{CONCLUSION}

Malocclusion is not just an invariable disease state, but a continuous spectrum of occlusal variation, occurring as a myriad of combinations and permutations of a number of heterogeneous traits or symptoms, each with its own wide range of severity and implications in creating a particular manifestation of occlusion.

In general prevalence of malocclusion is considered to be on increase with evolution and civilization. Although Angle's method has been used in recording the malocclusion it does not reflect the actual orthodontic treatment needs of the society. In India class II malocclusion has been reported to be relatively more prevalent in north Indian children population compared to southern India. However its prevalence in India is low compared to those reported in Caucasian population. The frequency of occurrence of class I malocclusion is greatest followed by class II and class III.

\section{REFERENCES}

1. Angle EH. Classificaiton of malocclusion. Dent Cosmos 1988;41:248-64.

2. Tang ELK, Stephen HY Wei. Recording and measuring malocclusion. A review of the literature. Am J Orthod Dentofac Orthop 1993;103:344-51.

3. McCall JO. A study of malocclusion in preschool and school children. Dent Items Interest 1944;131-33.

4. Björk A, Krebs AA, Solow B. A method for epidemiological registration of malocclusion. Acta Odontol Scand 1964;22:27-41.

5. Gravely LD, Johnson DB. Angles's classification of malocclusion: an assessment of reliability. $\mathrm{Br} \mathrm{J}$ Orthod 1974; 1(3):79-86.

6. Brzroukov V, Freer TJ, Helm S, Kalamkarov H, Sardoinfirri J, Solow B. Basic methods for recording malocclusion traits. Bull World Health Organ 1979;57(6):955-61.

7. Thilander B, Pena L, Infante C, Parada S Mayorga CD. Prevalence of malocclusion and orthododontic treatment need in children and adolescents in Bogota, Columbia: An epidemiological study related to different stages dental development. Eur J Orthod 2001;23:15367.

8. Shaw WC, Richmond S, O'Brien KD, Brook $P$, Stephens CD. Quality control in orthodontics: indices of treatment need and treatment standards. Br Dent $J$ 1991;170(3):107-12.

9. Evans R, Shaw W. Preliminary evaluation of an illustrated scale for rating dental attractiveness. Eur J Orthod 1987;9:31418.

10. Brook PH, Shaw WC. The development of an index of orthodontic treatment priority. Eur J Orthod 1989;11:309-20.

11. Kharbanda OP, Sidhu SS, Sundaram KR, Shukla DK. Occlusion status during early mixed dentition in Delhi children. Project Report Indian Council of Medical Research 1991.

12. Jacob PP, Mathew CT. Occlusal pattern study of school children (12- 15 years) of Tiruvananthapuram city. $J$ Indian Dent Assoc 1969;41:271-74.

13. Prasad AR, Shivaratna SC. Epidemiology of malocclusion a report of a survey conducted in Bangalore city. J Ind Orthod Soc 1971;3(3):43-55.

14. Rao DB, Hegde AM, Munshi AK. Malocclusion and orthodontic treatment need of handicapped individuals in South Canara, India Int Dent J 2003;53:13-18.

15. Sureshbabu AM, Chandu GN, Shafiulla MD. Prevalence of malocclusion and orthododontic treatment need among 13$15 y$ rs old school going children and adolescents of Davangere, Karnataka, India. J Indian Assoc Public Health Dent 2005;6:32-35.

16. Das UM, V Reddy D. Prevalence of malocclusion among school children in Bangalore, India. Int J Clin Ped Dent 2008;1:10-12.

17. Shivakumar KM, Chandu GN, Subba Reddy VV, Shafiulla MD. Prevalence of malocclusion and orthodontic treatment needs among middle and high school children of Davangere city, India by using Dental Aesthetic Index. J Indian Soc Pedod Prev Dent 2009;27:211-18.

18. Suma G, Usha Mohan Das. Crowding, spacing and closed dentition and its relationship with malocclusion in primary dentition. IJDS 2010;1(1):16-19.

19. Kharbanda OP. Orthodontics-diagnosis and management of malocclusion and dentofacial deformities. Mosby Elsevier. First edition. 2009;20-26.

20. Joshi MR, Makhia PG. Some observation on spacing in normal deciduos dentition of 100 Indian children from Gujrat. $\mathrm{Br} J$ Orthod 1984;11:75-79.

21. Singh SP, Utreja A, Chawla HS. A study of distribution of malocclusion among north Indians seeking orthodontic treatment. J Ind Orthod Soc 1993;24(2): 47-53.

22. Kharbanda OP. Sidhu SS. Prevalence studies on malocclusion in India retrospect and prospect. $J$ ind Orthod Soc 1993;24(4):115-18.

23. Kharbanda OP, Sidhu SS, Sundaram KR, Shukla Dk. A study of malocclusion and associated factors in Delhi children. $J$ Pierre Fauchard Academy 1995;9:7-13.

24. Kharbanda OP, Sidhu SS, Sundararn KR, Shukla DK. Prevalence of malocclusion and its traits in Delhi children. J Indian Orthod Soc 1995;26(3):98-103.

25. Singh A, Singh B. Kharhancda OP, Shukla DK, Goswami K, Gupta S. Malocclusion and its traits in rural school children from Haryana. J Ind Orthod Soc 1998;31:7680.

26. Jalili VP, Sidhu SS, Kharhanda OP. Status of malocclusion in Tribal children of Mandu (central India). J Ind Orthod Soc 1993;24:41-46.

27. Trehan M, Chugh VK, Sharma S. Prevalence of malocclusion in Jaipur, India. IJCPD 2009;2(1):23-25.

28. Khandelwal A, Jalili VP, Jain S. Incidence of malocclusions in males of Indore, Malwa. JIDA 2010;4(10):357-58.

29. Jarvinen $S$, Vaataja P. Variability in assessment of need for orthodontic treatment when using certain treatmentneed indices. Community Dent Oral Epidemiol 1987;15:245-48. 